\title{
Information-seeking in the brain
}

\author{
Caroline J. Charpentier ${ }^{1,2 *}, \mathrm{PhD} \&$ Irene Cogliati Dezza ${ }^{3,4,5 *}, \mathrm{PhD}$ \\ ${ }^{1}$ Division of Humanities and Social Sciences, California Institute of Technology \\ ${ }^{2}$ Institute of Cognitive Neuroscience, University College London \\ ${ }^{3}$ Department of Experimental Psychology, Faculty of Brain Sciences, University College London \\ ${ }^{4}$ The Max Planck UCL Centre for Computational Psychiatry and Ageing Research, University College \\ London \\ ${ }^{5}$ Department of Experimental Psychology, Ghent University.
}

*These authors contributed equally

\begin{abstract}
$\underline{\text { Abstract }}$
Recent advancements in psychology, behavioral economics and neuroscience have shown the human pursuit of knowledge to be an essential aspect of human cognition. It drives intellectual development, is integral to social interactions, and is crucial for learning, decision-making and goal-directed behavior. Information appears to be valuable in and of itself, even when it has no apparent use, whereas at other times, instrumental information is actively and paradoxically avoided. With this complex role, a wide range of neural mechanisms can be deployed to assign value to information and drive decisions to seek (or avoid) information. Evidence points towards key roles for the mesolimbic system and the prefrontal cortex in these processes. Specifically, two different networks appear to be involved in the implementation of information-seeking behaviors. One network, overlapping with areas involved in processing primary and monetary rewards, appear to drive a general preference for information, as well as valence-dependent information-seeking. The other network, independent of reward processing, is recruited when information is acquired to reduce uncertainty. In this chapter, we review some of the most recent discoveries in the field to provide an overview of the neural basis of information-seeking.
\end{abstract}




\section{Introduction}

Humans exhibit a strong preference for information (Gottlieb, Oudeyer, Lopes, \& Baranes, 2013) and spend a substantial amount of time seeking information (e.g., asking questions, reading, internet browsing). In our modern connected societies, we are very often bombarded with information, coming from different sources and with different levels of veracity. As a result, we have to actively weigh the pros and cons of seeking information (versus refraining from doing so), depending on what we expect information will provide us with (e.g., good or bad news, better understanding of reality etc.).

Information is needed to guide people's decisions and actions, but it is also necessary to better comprehend and predict the physical and social world. Information may also satisfy an internal need such as a desire for novelty (Gottlieb et al., 2013), curiosity (Kidd \& Hayden, 2015), or uncertainty reduction (Gershman, 2019). Additionally, information can impact the current mood and affective states of the information-seeker (Sharot \& Sunstein, 2020). For example, receiving information about a positive HIV test result may induce sadness, while receiving a job offer may induce joy. As such, information-seeking is a complex behavior which drives intellectual development, is integral to social interactions (Evans \& Chi, 2010; Evans, Kairam, \& Pirolli, 2009), and is crucial for learning, decision-making and goal-directed processing (Gottlieb et al., 2013).

Given the complexity associated with the human pursuit of knowledge, understanding its underlying mechanisms is inherently challenging. Many motives (see Table 1 for definitions) can play a role in driving information-seeking (e.g., satisfy internal or external needs, as described above and in several other chapters in this book such as Chapter 1, 2,10). These motives also influence individual expectations during the anticipation of information, as well as the goals that can be achieved if the information is consumed (e.g., impact on internal or external states). Only recently in the information-seeking field, researchers have started to better characterize these behaviors (Zurn \& Bassett, 2018; Kobayashi, Ravaioli, Baranes, Woodford, \& Gottlieb, 2019; Murayama, 2019a; Sharot \& Sunstein, 2020), as well as their underlying computational and algorithmic principles (Cogliati Dezza, Yu, Cleeremans, \& Alexander, 2017; K. J. Friston et al., 2017; Oudeyer, 2018; Wu, Schulz, Speekenbrink, Nelson, \& Meder, 2018; Schulz \& Gershman, 2019; Dubey \& Griffiths, 2020).

As a consequence, understanding how the brain implements information-seeking is still in its early stages. Moreover, the experimental paradigms and computational frameworks that have been used thus far to understand the neurocognitive processes associated with information-seeking have varied widely across studies. Some studies have used 'observing' paradigms, whereby information is clearly non-instrumental (Bromberg-Martin \& Hikosaka, 2009; Bromberg-Martin \& Hikosaka, 2011; Blanchard, Hayden, \& Bromberg-Martin, 2015; Charpentier, Bromberg-Martin, \& Sharot, 2018), allowing researchers to isolate how information is valued in and of itself, as well as the role of specific non-instrumental motives. Other studies have instead focused on both instrumental and non-instrumental motives (Kobayashi \& Hsu, 2019; Cogliati Dezza, Cleeremans, \& Alexander, 2020) or have quantified information-seeking behavior through measuring and modelling exploratory behavior during learning (e.g. explore-exploit dilemma, foraging problems; Wilson, Geana, White, Ludvig, \& Cohen, 2014; Cogliati Dezza et al., 2017; Gershman, 2019). In exploratory paradigms, for example, the information gathered is instrumental, since it helps improve future actions and outcomes, but it also helps to better understand the environment. In this context, it is much harder to dissociate the specific drivers and mechanisms of information-seeking, since motives may be more confounded (unless computational modelling techniques are adopted; Cogliati Dezza et al., 2020). Overall, it is important to highlight that the level of variability in experimental approaches for what is a relatively nascent field, makes it quite difficult to interpret current findings within a fully coherent picture.

Nevertheless, recent advances in the neuroscience of information-seeking all seem to converge on a key principle: information is valuable, similar to primary or monetary rewards (Murayama, 2019a). Humans and other animals are oftentimes willing to incur a cost for obtaining information (Pierson \& Goodman, 
2014; Blanchard et al., 2015; Charpentier et al., 2018), even if the information provides no or few instrumental benefits. This is reflected in the brain via a common neural code for reward and information (Kobayashi \& Hsu, 2019), which seems to be a rather universal mechanism across many informationseeking behaviors, encompassing general preference for information, as well as information driven by instrumentality or valence. However, when people seek information to better understand the surrounding environment and reduce their uncertainty about the world, information-seeking appears to be implemented in the brain through distinct neural circuits. This idea of dissociable networks is consistent with a recent review suggesting distinct roles for anterior and posterior regions of the prefrontal cortex in informationseeking processes (Kaanders, Juechems, O’Reilly, \& Hunt, 2021).

This chapter will review the main findings from the field, which show both shared and independent networks underlying how information-seeking is implemented in the brain. We will then conclude the chapter by presenting a possible mechanism based on recent theories (Bromberg-Martin \& Monosov, 2020; Murayama, 2019a; Sharot \& Sunstein, 2020), which suggests that the neural mechanisms of informationseeking are controlled by a unique network, with some mechanisms shared with reward processing and others independent of it.

\begin{tabular}{|c|l|}
\hline Information-seeking motives & \multicolumn{1}{c|}{ Definition } \\
\hline Instrumentality & $\begin{array}{l}\text { The potential of information to improve future decisions, actions, and } \\
\text { outcomes. Preference for/value of information increases the more } \\
\text { 'useful' the resulting knowledge is. }\end{array}$ \\
\hline Curiosity & $\begin{array}{l}\text { The potential of information to satisfy an internal drive for } \\
\text { knowledge. Preference for/value of information increases when } \\
\text { subjective desire to know something is high. }\end{array}$ \\
\hline Valence & $\begin{array}{l}\text { The potential of information to influence mood and affective states. } \\
\text { Preference for/value of information increases with the desirability of } \\
\text { the news provided. }\end{array}$ \\
\hline Uncertainty reduction & $\begin{array}{l}\text { The potential of information to reduce uncertainty in the environment } \\
\text { and to increase understanding of the world. Preference for/value of } \\
\text { information increases when uncertainty or entropy is high. }\end{array}$ \\
\hline
\end{tabular}

Table 1. Information-seeking: a multifaceted process. Many motives can drive information-seeking behavior, making it a complex process to study. As shown in the table information can improve people's actions and decisions (instrumentality), it can satisfy an internal drive for knowledge (curiosity), affective states (valence) and the understanding of reality (uncertainty reduction). Definition for each of these motives are provided in the table.

\section{Shared neural correlates for information and reward processing: a domain-general preference for information?}

A large body of literature that has examined how the brain processes information has converged on the finding that the opportunity to gain information relies on the same neural circuitry as the opportunity to gain rewards (Figure 1A).

Considerable empirical evidence in neuroeconomics and decision neuroscience suggest that when learning and assigning value to outcomes, the brain relies on a prefrontal-mesolimbic network (Bartra, McGuire, \& Kable, 2013; Lopez-Persem et al., 2020). Key regions of this network include the ventromedial prefrontal cortex (vmPFC), orbitofrontal cortex (OFC), ventral striatum and dopaminergic midbrain (substantia nigra and ventral tegmental area - SN/VTA). Activity in prefrontal regions tends to reflect the economic value of decision options as well as the difference in value prior to a choice (Bartra et al., 2013; Pessiglione \& 
Lebreton, 2014; Padoa-Schioppa \& Conen, 2017). In parallel, activity in SN/VTA and ventral striatum updates the expected value of an outcome by computing a prediction error. This signal is reflected in dopaminergic neurons, which fire up when an outcome is better than expected and decrease their firing rate when outcomes are worse than expected (Schultz, Dayan, \& Montague, 1997; Diederen \& Fletcher, 2021). We note, however, that these regions are extensively connected with each other, both anatomically and functionally (Goldman-Rakic P.S., 1992; Chib, Yun, Takahashi, \& Shimojo, 2013; Morris et al., 2016), and thus reward-related signals are encoded across the entire network more so than within each single region independently.

Interestingly, recent findings in the literature suggest that each of these regions also play a role in encoding the opportunity to gain information, independent of its reward value (Figure 1A).

First, the OFC has been implicated in representing information gain, both at the single-neuron levels in nonhuman primates, and in the BOLD signal in humans. In a seminal study (Blanchard et al., 2015), monkeys made sequential choices between two gambles displayed on the left and right sides of the screen. One gamble was informative as it always led to a cue which predicts the gamble's outcome. The other gamble led to a non-informative cue. OFC neural populations were found to use different neural codes to signal the opportunity to gain information about rewards versus the value of the rewards themselves. In other words, information and rewards were signaled with independent neural codes in OFC neurons. Interestingly, this distinct encoding of reward and information allowed for expectations about reward and information values to be adjusted separately depending on internal drives, which in turn influences decisions and actions. For example, it would allow an agent to seek food when hungry, but to seek information when uncertain. In human fMRI, the lateral OFC was also found to represent informational signals (Jessup \& O'Doherty, 2014), which encode whether an outcome is rewarding or punishing (compared to a neutral outcome), regardless of its valence, utility, or magnitude. More recently, a cluster in the medial portion of OFC was found to exhibit increased activity during the presentation of cues that predict the upcoming delivery of information, relative to the presentation of ignorance cues signaling that no information would be delivered (Charpentier et al., 2018). Importantly this signal was again independent from the valence of the information (i.e., whether the information was about a reward, a loss, no reward, or no loss). This suggests that the OFC plays a possible role in signaling a general preference for knowledge over ignorance, in both human and non-human primates.

Another line of work has also provided evidence for a role of the dopaminergic system, specifically lateral habenula (LHb), SN/VTA, and nucleus accumbens (a portion of the ventral striatum), in general information preference. Bromberg-Martin and Hikosaka (2009) recorded from macaques' midbrain dopamine neurons while the animals performed a task in which they viewed either informative cues (cues that deterministically predict whether a small or a large reward is coming) or non-informative cues (random cues that each lead to the small or the big reward with 50\% chance). Prior to these cues, the animals either chose from or were passively presented with one of two other cues, which this time predicted whether or not information (i.e., the cues that deterministically predict the reward) would be shown next. Behaviorally, the animals exhibited a strong preference for receiving advance information about the size of their reward. This preference was reflected in the response of midbrain dopamine neurons, which were found to signal the expectation of receiving information. Importantly, contrary to the OFC neurons identified in the Blanchard et al.'s study, which signaled reward independently from information (Blanchard et al., 2015), here, those same midbrain dopamine neurons that signaled expected information were found to signal expected reward, suggesting a common neural code in dopamine neurons. In humans, consistent with the idea of a shared neural code, fMRI BOLD responses in the nucleus accumbens encoded two properties of the outcome (Smith, Rigney, \& Delgado, 2016): the affective component (number of points earned) and the informative component (amount of knowledge gained from feedback). Additionally, the strength of the informative - but not affective - signal predicted subsequent performance in using the information to obtain rewards, suggesting a role for the nucleus accumbens in processing instrumental information. Similarly, in a recent study (Filimon, Nelson, Sejnowski, Sereno, \& Cottrell, 2020), the nucleus accumbens 
was found to respond not only to the valence of feedback (positive versus negative), but also to the expectation of a more informative stimulus (high versus low information to help stimulus categorization).

Thus, evidence in both human and non-human primate studies point towards a clear role of the brain's reward system in processing the value of information. Yet the specific computations that underlie this shared neural code remain unclear. Several studies provide some initial key insights into these possible computations. Similar to reward prediction errors, which rely on the dopaminergic system and allow learning about rewards in the environment, an early theory posited that the dopaminergic system may also encode an information prediction error (IPE), that is, the difference between the delivery or denial of information and the initial expectation of information. Following up on their earlier work, Bromberg-Martin and Hikosaka (2011) modified their task design in order to be able to quantify and detect the presence of information prediction errors. That is, they added the presence of an initial cue, which led to the delivery of informative cues vs. non-informative cues $50 \%$ of the time. This allowed for the comparision of neural responses for expected information vs. unexpected information. Recording from the lateral habenula ( $\mathrm{LHb})$, which contains dopamine neurons and encodes reward prediction errors (Matsumoto \& Hikosaka, 2007), they found that the neurons encoded an information prediction error (IPE). Specifically, LHb neurons responded more strongly when the delivery of informative or non-informative cues was unexpected compared to when it was expected. In humans, fMRI evidence for a domain-general IPE is still lacking, however, a study using EEG (Brydevall, Bennett, Murawski, \& Bode, 2018) found that feedback-related negativity signals when informative cues are revealed can represent both an information prediction error and a reward prediction error. While difficult to assess whether these two signals come from the same source, this is nonetheless an additional piece of evidence for a similarity in the type of neural signal the brain uses to represent reward and information. In other words, given EEG evidence only, the source could be different, but the computational code (prediction error) appears to be the same for reward and information. With additional evidence coming from monkey recording studies and human fMRI studies, current findings seem to converge towards the theory that both the source and the code of neural signals are shared between reward and information processing.

The most convincing evidence for shared neuro-computational signals for information and reward in the human brain comes from a recent study (Kobayashi et al., 2019), which examined both vmPFC and ventral striatum in human decisions to buy information about the true probability associated with a lottery. Activity in both regions correlated with the subjective value of information (SVOI), a trial-by-trial variable from a computational model that integrates the influence of both instrumental and non-instrumental motives on information-seeking decisions. That is, the more valuable information is to an individual, the stronger vmPFC and ventral striatum responses will be. Using multivariate analysis, the authors additionally showed that a decoder trained on reward-related responses (specifically, the expected utility signal; EU) could predict information-related responses (SVOI signal) and vice-versa. This suggests a common code for the subjective value of information (SVOI) and for the expected utility of the lotteries (EU). While the aforementioned SVOI signal did not separate the contribution of instrumental and non-instrumental information (rather reflecting the integration of the two), the finding of a recent study (Cogliati Dezza et al., 2020) suggests, albeit in a different task, that it is the instrumental value of information that is expressed in reward regions. This study additionally shows the convergence of reward and information signal in the striatum region, consistent with (Smith et al., 2016).

In summary, this body of literature has led to the now widely accepted assumption that information is intrinsically rewarding. The shared neural correlates described above, provide a possible explanation for why individuals, both humans and other animals, exhibit a strong preference for information, and why they are oftentimes willing to incur a cost for obtaining such information, even when it provides no or few instrumental benefits. In the next sections, we will focus on the neural mechanisms for two main motives of these information-seeking decisions: valence and uncertainty reduction. 
A

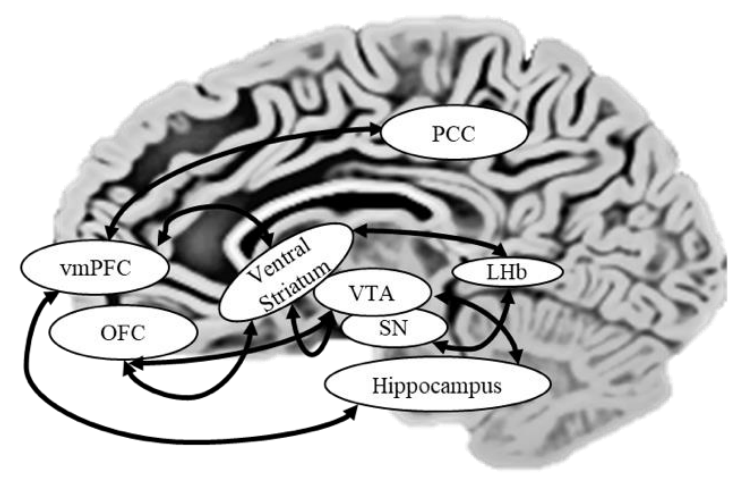

B Independent network

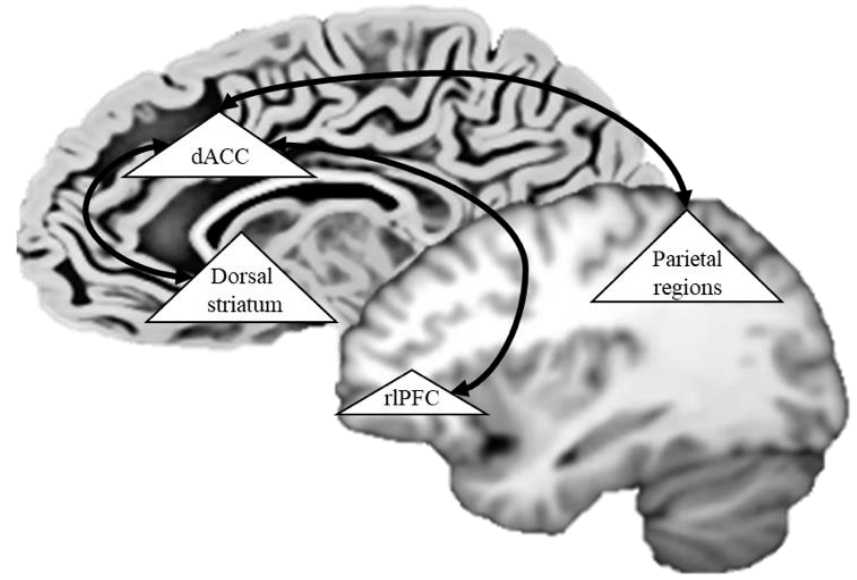

Figure 1. Two main brain networks are recruited during information-seeking. Here we hypothesize some connections based on anatomical and functional connectivity usually found in the literature among the regions involved in information-seeking. However, the function and directionality of these connections is mostly unknown. A) A prefrontal-mesolimbic 'shared' network appears to encode both the value of information and the value of primary and monetary rewards, as well the effect of valence on informationseeking. B) Uncertainty reduction, another motive for information-seeking, is represented in an 'independent' fronto-parietal network.

\section{Valence-dependent information-seeking: neural mechanisms of the preference for good news}

While the opportunity to gain information carries value in and of itself, independent of the valence of the information, studies have demonstrated a clear bias whereby information likely to induce positive affect (desirable information) is preferred over information likely to induce negative affect (undesirable information; Sharot \& Sunstein, 2020). Note that this valence-dependent information preference is present even when information has no instrumental use, i.e., when it cannot be used to influence one's outcomes (Charpentier et al., 2018; Kobayashi et al., 2019). In other words, an individual is more likely to open an envelope containing the results of a medical test if they expect the result to be good than if they expect it to be bad. This preference can be explained by belief utility theory (Bénabou, 2016; Loewenstein \& Molnar, 2018), which is a theory from behavioral economics positing that our beliefs (e.g., the belief that my medical test result will carry good news), as well as the ability to anticipate the experience of outcomes, have utility, similar to goods or monetary rewards. Therefore, seeking desirable over undesirable information allows one to maintain positive belief utility by anticipating positive future outcomes (Loewenstein, 1987; Caplin \& Leahy, 2001). This is thought to contribute to higher mood and well-being. Dread, on the other hand, would lead to negative belief utility (Berns et al., 2006 Story et al., 2013), and to the tendency to avoid undesirable information (Karlsson, Loewenstein, \& Seppi, 2009; Golman, Hagmann, \& Loewenstein, 2017). For example, by leaving the envelope unopened if I expect the medical test result to carry bad news.

One possible mechanism for such valence-dependent information-seeking behavior is through the computation of valence-dependent information prediction errors (VD-IPE). This represents an IPE signal (i.e., error in predicting the opportunity to receive information) that scales with the desirability of the outcome. In Charpentier et al. (2018), participants had to decide between a high and a low probability of receiving information about the outcome of a probabilistic lottery. Behaviorally, participants' propensity to choose the high information probability increased with the probability of winning the lottery and decreased with the probability of losing the lottery. This valence-dependent information-seeking behavior was 
mirrored in the brain by VD-IPEs, which were detected in the SN/VTA, such that IPEs were represented more strongly in the gain domain than in the loss domain. In the nucleus accumbens - one of the regions SN/VTA dopaminergic neurons project to - the strength of the VD-IPE signal predicted the effect of valence on participants' information-seeking decisions. In other words, the more IPE signals differed for desirable versus undesirable information, the more participants' decisions to seek information were biased by the valence of the lottery.

Another mechanism contributing to the influence of hedonic motives on the valuation of information involves the notion of savoring, that is, the propensity for advanced knowledge of an upcoming reward to increase our anticipation of it. Computationally, a mechanism was proposed for this process (Iigaya, Story, Kurth-Nelson, Dolan, \& Dayan, 2016), such that the anticipatory utility associated with knowledge of a future outcome is boosted by the reward prediction error (RPE). In a recent neuroimaging study, Iigaya et al. (2020) proposed that a network including vmPFC, hippocampus and midbrain regions underlies this boosting of anticipatory utility by RPEs. Specifically, vmPFC tracks the value of anticipatory utility, the SN/VTA and medial posterior cingulate cortex (mPCC) represent information that enhances anticipation (the anticipatory RPE), and the hippocampus encodes the absolute anticipatory RPE or surprise (independent of valence). These three regions were found to be functionally connected, such that the hippocampus-SN/VTA connectivity was modulated by the anticipatory utility predicted by the model, and the hippocampus-vmPFC connectivity was modulated by the anticipatory RPE. In addition to the typical reward processing areas, the hippocampus appears to play an integral role in integrating the effect of valence (here RPE) on information (here anticipatory utility). This study is also noteworthy for examining temporal aspects of the fMRI signals, with vmPFC activity found to be ramping up in anticipation of a reward in a way that is boosted by the surprise (RPE) associated with the information, rather than simply ramping up proportionally to the reward expected value. This finding raises the intriguing possibility of a bi-directional interaction between reward and information. Not only can expected reward increase information preference, but unexpected information can increase reward anticipation in the brain.

An obvious question following from this line of work is whether dopamine plays a role in underlying hedonic motivations during information-seeking. In a recent pharmacology study, administering L-DOPA (a precursor to dopamine) or a placebo to human participants (Vellani, de Vries, Gaule, \& Sharot, 2021), L-DOPA was actually found to eliminate the valence bias that is typically observed during informationseeking. Specifically, participants under L-DOPA preferred information equally for gains and losses. The difference was specifically driven by an increased preference for information about losses in the L-DOPA group relative to the placebo group. A possible mechanistic explanation for this finding is that L-DOPA administration may help reducing the anticipated negative affect or dread associated with the anticipation of a loss. Therefore, dopamine may influence how expected negative outcomes are processed in the midbrain or ventral striatum, in turn making information about these outcomes less aversive. Using ${ }^{18} \mathrm{~F}-$ FDOPA PET scanning to quantify individual dopamine synthesis capacity, Van Lieshout et al. (2020) replicated the behavioral finding that curiosity increases as a function of outcome valence (i.e., higher for expected gains than for expected losses). Yet they found no evidence that individual differences in curiosity or the sensitivity to outcome valence was related to dopamine synthesis in ventral striatum, caudate or putamen. This suggests that more work is needed to examine the role of dopamine in valence-dependent information seeking.

In summary, the influence of valence on information-seeking appears to also rely on neural mechanisms shared with reward processing, as those identified in the first part of the chapter. This shared network between reward and information, as we have discussed, is assumed to play a role in assigning value to the opportunity to gain information, independently of the valence of the information. However, it is perhaps unsurprising that outcome valence, which is known to be represented within this same network, would recruit these same mesolimbic and prefrontal regions to influence the value of information (Figure 1A). While the existing (yet limited) literature on this topic suggests converging evidence for a role of the reward, and possibly dopaminergic, systems in valence-dependent information-seeking, several mechanistic 
accounts have been proposed and further investigations are needed to paint a more coherent picture of the exact computations performed by these brain regions.

\section{Uncertainty reduction: an independent information-seeking network}

While humans seek information that makes them feel good or improves future actions, they also seek information that helps them to better understand the world by reducing subjective uncertainty. Uncertainty reduction is therefore a key motivational factor to seek information (Berlyne, 1957; Gershman, 2019; Sharot $\&$ Sunstein, 2020). Different parameterizations of uncertainty reduction are often added in computational models for human cognition and behavior or in artificial agents to motivate information-seeking (Wilson et al., 2014; Oudeyer, Lopes, Kidd, \& Gottlieb, 2016; Cogliati Dezza et al., 2017; Gershman, 2018; Schwartenbeck et al., 2019). This drive continues to prevail even in situations where information is noninstrumental (Kobayashi et al., 2019; White et al., 2019) and is linked to an intrinsic motivation to reduce entropy in a system (Oudeyer \& Kaplan, 2007; Friston et al., 2015).

While both instrumental and valence-dependent motives for seeking information mostly recruit brain networks typically involved in reward processing (Figure 1A), the motivation to reduce uncertainty when seeking information seems to rely on a separate and independent neural network (Figure 1B). This independent network includes regions of the prefrontal cortex, such as dorsal anterior cingulate cortex (dACC) and rostrolateral prefrontal cortex (rlPFC) as well as portions of the parietal cortex and the dorsal striatum. In what follows, we present studies which have individuated key nodes of this network. It is worth mentioning that information-seeking driven by uncertainty and exploration often overlaps in the literature. For the sake of this chapter, however, we only focus on exploration findings in which uncertainty reduction is explicitly identified as the drive for exploration, in contrast with exploratory behaviors that are driven by randomness in the learning or decision process.

Evidence for the involvement of dACC in processing information-seeking motivated by uncertainty reduction comes from recent studies on humans (Cogliati Dezza et al., 2020; Kaanders, Nili, O'Reilly, \& Hunt, 2020) and monkeys (Hunt et al., 2018; White et al., 2019). A first study conducted on human subjects highlighted the existence of independent systems for information and reward in the prefrontal cortex (Cogliati Dezza et al., 2020). In particular, human subjects were asked to make sequential choices among 3 decks of cards. Cards varied in the level of information (i.e., how much the subject was ignorant about an option due to prior experience) and reward. In this task, subjects sought information about options they were more uncertain about (non-instrumental information), even in the absence of an instrumental benefit to seek information. Subsequent fMRI analysis showed that dACC and anterior insula encoded the noninstrumental value of information after controlling for the variance explained by the reward value of an option and the instrumental value of information. On the contrary, vmPFC and PCC encoded the reward value of the chosen option after controlling for the variance explained by non-instrumental information. This study identifies regions in the brain, including dACC, which encode the non-instrumental value of information independently of the reward value associated to the options. The involvement of dACC in information processing has also been shown in a second fMRI study, in which a portion of the medial frontal cortex (encompassing dACC and pre-supplementary motor cortex) was found to encode the willingness to sample information to reduce reward uncertainty (Kaanders et al., 2020). Evidence for the involvement of dACC in information processing comes also from non-human primate studies. For example, White et al. has identified an intricate network, including ACC and dorsal striatum, which specifically signals information-seeking but not reward-seeking (White et al., 2019). In particular, researchers presented monkeys with visual conditioned stimuli predicting reward information (i.e., 100\%, 50\% and 0\%). ACC neurons (alongside neurons in the dorsal striatum) were strongly activated when the cue predicting reward was uncertain, with increased activation until information to resolve uncertainty was delivered. The strength of this ramping caused gaze to shift towards information-related cues before receiving information to 
resolve uncertainty, and was specifically related to anticipating information and not to reward value or uncertainty per se. ACC neurons were the earliest predictor of gaze shifts. This suggests that ACC may have a supervisory role in sustaining information-seeking. A similar role for ACC in sustaining informationseeking was observed in an additional study (Hunt et al., 2018), in which monkeys' ACC neurons, primarily recorded in the dorsal bank of the cingulate sulcus, tracked how each piece of information reduced the uncertainty associated with the chosen action.

Another region involved in information-seeking driven by uncertainty reduction is the rlPFC. This region seems to track the amount of surprise humans recently experienced in a given context (Ligneul, Mermillod, \& Morisseau, 2018). Additionally, rlPFC encodes trial-by-trial changes in relative uncertainty, which increases exploration for uncertain options (Badre, Doll, Long, \& Frank, 2012; Tomov, Truong, Hundia, \& Gershman, 2020). In particular, Badre et al. (2012) asked participants to stop a rotating clock to win points. To maximize rewards, participants needed to learn the optimal speed (either "fast" or "slow" responses) Computational models were then adopted to compute the relative uncertainty associated with response time, with the assumption that subjects explore uncertain response times to reduce their uncertainty. Interestingly, rlPFC not only tracked trial-by-trial changes in relative uncertainty associated with exploratory decisions, but this pattern distinguished individuals who were using uncertainty as a drive for exploration from those who did not. A later study (Tomov et al., 2020) showed that activity in rlPFC cortex correlates with exploration to seek information (i.e., directed exploration), while activity in dorsolateral prefrontal cortex was associated with a different type of exploration (i.e., random exploration; (Wilson et al., 2014). It is worth noticing that other regions may play a role in processing directed exploration including the frontopolar cortex (Zajkowski, Kossut, \& Wilson, 2017).

Another important region involved in information-seeking driven by uncertainty reduction is the parietal cortex. Van Lieshout et al. (2018) showed that humans were particularly curious when information had a greater impact in reducing subjective uncertainty, and this increase in uncertainty-driven curiosity was associated with BOLD responses in the parietal cortex. Parietal neurons in monkeys appear to also encode information gain based on changes in uncertainty (Horan, Daddaoua, \& Gottlieb, 2019), as well as how much information reduces uncertainty (Foley, Kelly, Mhatre, Lopes, \& Gottlieb, 2017). Interestingly, parietal neurons were specifically encoding information-related signals but not reward-related signals. Similar results were found in human subjects where bilateral activation of intraparietal sulcus was found to encode the willingness to sample additional information in order to reduce uncertainty about rewards (Kaanders et al., 2020), suggesting a role of the parietal cortex in encoding uncertainty-driven informationseeking.

In summary, when humans decide to seek information to reduce uncertainty, a network comprised of the dACC, dorsal striatum, rlPFC and parietal cortex intervenes at different stages of the process. Key nodes of this network are also consistent with the animal literature. While uncertainty reduction seems to rely on an independent network compared to other information-seeking drives, more research is needed to identify the reciprocal connections between key nodes of this network.

\section{Conclusion}

Information-seeking is a complex behavior. To be implemented, it requires a wide range of neural circuitry. General preference for information but also how valence influences this preference, with both appearing to rely on similar neural mechanisms to those involved in processing primary or monetary rewards. A recent review (Bromberg-Martin \& Monosov, 2020) proposed a hypothetical network for information-seeking, suggesting that the value of information and the value of rewards are encoded in the same brain areas (OFC \& ACC, projecting to striatum and pallidum) but in different neurons, thus allowing for the separate pursuit of information and rewards depending on individual motivations and the environment. These two value signals would then be integrated in striatal and mesolimbic areas, possibly relying on dopaminergic 
neurons, to compute a total value signal integrating both information prediction and reward prediction (Cogliati Dezza et al., 2020). In parallel, an independent network, recruiting dACC, rlPFC and parietal regions, seems to drive information-seeking to reduce uncertainty. To date, however, how uncertainty reduction signals could also be integrated into the computations of value signals discussed above remain to be established.

A novel theory (Sharot \& Sunstein, 2020) and recent empirical evidence (Kelly \& Sharot, under review; Cogliati Dezza \& Sharot, in prep) point toward a joint influence of instrumental utility, valence and uncertainty reduction in motivating information-seeking. In other words, when humans decide to seek information, the value of information (or information prediction) is computed as the weighted sum of different motives. It is therefore possible that some level of interactions among these networks occurs when humans decide to seek information. This would suggest that the neural mechanisms of information-seeking are rather controlled by a unique network, with some mechanisms shared with reward processing and others independent of it.

In Figure 2, we describe a possible mechanism for how the "overall information-seeking network" may operate. Expectations for uncertainty reduction, valence, and instrumental utility are computed in distinct brain regions. These expectations may then be integrated in the OFC to compute the information prediction which then guides behavior. While the OFC seems to be involved in processing information predictions, it is still unknown whether it also computes the weighted sum of the three motives (Sharot \& Sunstein, 2020), or instead, if other regions are involved in this process. If the resulting behavior is to seek information, an information feedback is provided and used to update the information expectations computed in the brain. It is worth noting that the neural mechanisms by which the three motives are updated are still unclear. A recent framework suggests that knowledge can be learned and updated following similar reinforcement learning mechanisms as those used to learn about rewards (Murayama, 2019b, and chapter 2 of this book). And, neural evidence suggests that the brain computes information prediction error (IPE; Bromberg-Martin \& Hikosaka, 2011) and valence-dependent IPE (VD-IPE; Charpentier et al., 2018). However, how these information prediction errors interact with the value associated with each motive is still unknown.

Several other substantial questions remain unanswered in this nascent field of information-seeking neuroscience.

First, there are very few causal studies so far examining how necessary these networks, and the regions within them, are for driving information-seeking decisions. Preliminary evidence involve a possible causal role for dopamine in motivating information-seeking about negative outcomes (Vellani et al., 2021), for the rlPFC in information-driven exploration (Zajkowski et al., 2017), and for the basal ganglia (dorsal striatum and pallidum) on the speed at which information is acquired (White et al., 2019). Yet, the causal involvement of some of the key regions of our proposed network, such as the vmPFC, ventral striatum, dopaminergic midbrain, dACC or parietal regions, has not been investigated at this point. Future pharmacology or lesion studies are needed to address this gap.

Second, whether and how the dynamics of information-seeking are represented in the brain is an open question. In other words, how does the brain track and updates the value of information over time in order to adapt its decisions to seek or avoid information? One study provides preliminary evidence that the dlPFC, a region not implicated so far in the processes described in this chapter, may be involved in this dynamic process of tracking the value of information over time (Kobayashi et al., 2021). How this region is integrated in the information-seeking network will hopefully be examined in more details in future studies.

Lastly, the neurochemical mechanisms underlying the information-seeking network(s) remain to be established. Recent converging evidence appears to suggest an involvement of dopamine not only in valence-dependent information-seeking (Vellani et al., 2021), but also in uncertainty-driven exploration (Frank, Doll, Oas-Terpstra, \& Moreno, 2009). An intriguing possibility is that dopamine signals could play a role in tying the "overall information-seeking network" together, and strengthening the interaction 
between motive-specific processes, such as valence and uncertainty reduction. Further evidence is needed to test this possibility as well as to identify the role of other neurotransmitters such as serotonin and norepinephrine in information-seeking behaviors.

To sum up, recent advances in neuroscience all converge on the notion that information is intrinsically valuable. Some information-seeking behaviors share indeed a common neural code with reward processing and general valuation mechanisms. However, when people seek information to reduce uncertainty a different route of implementation seems to be taken. These different routes could actually provide very useful bottom-up constraints to help refine our understanding of the algorithmic and computational implementation of information-seeking, which in turn will help to better characterize the neural mechanisms underlying information-seeking.

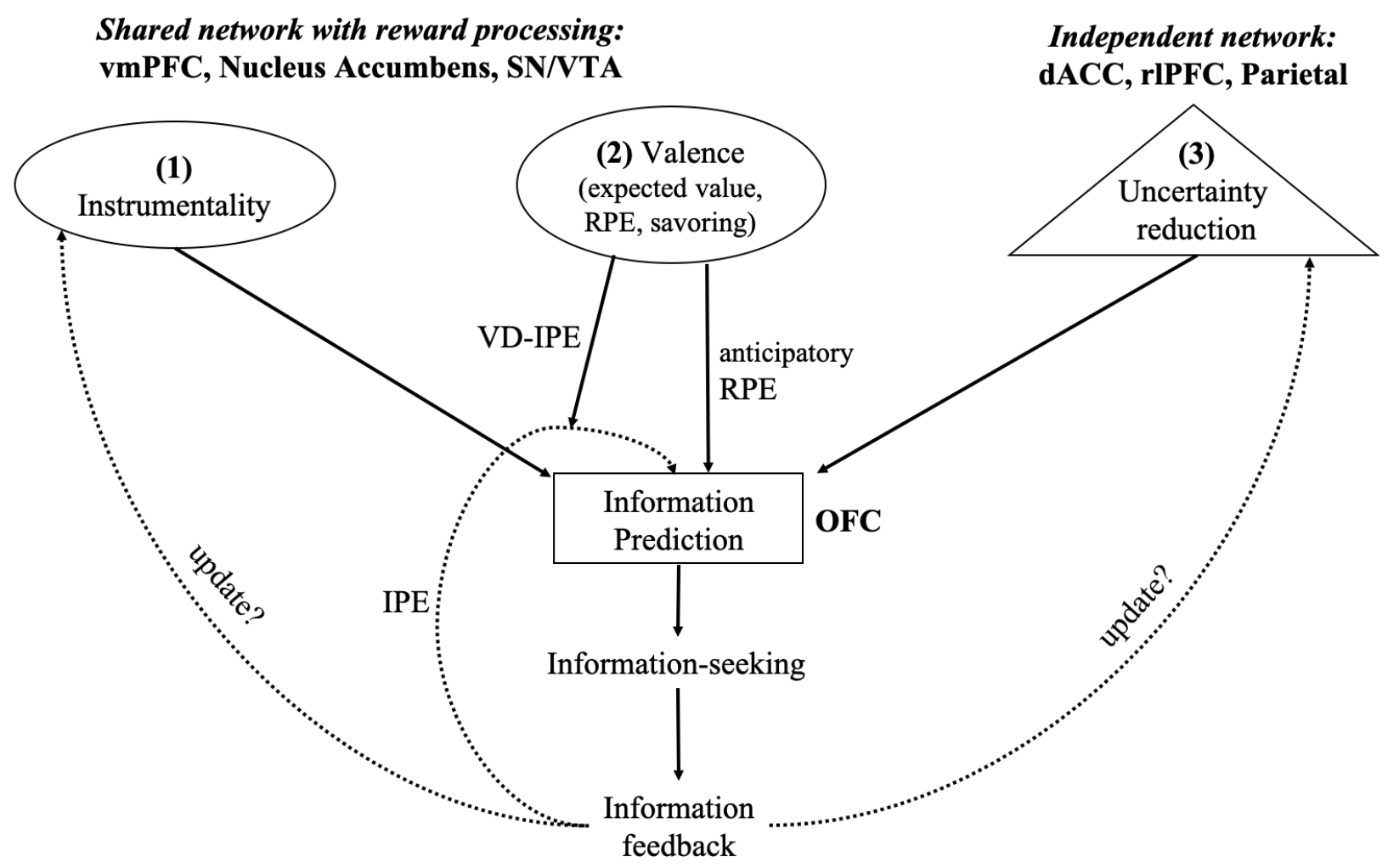

Figure 2. Overall information-seeking network. According to this hypothesized integrated network, information-seeking motives (instrumentality, valence, uncertainty reduction) are computed in distinct regions across the brain, and influence information predictions. While these regions probably interact in ways that are yet to be established, current evidence suggests that (1) the vmPFC may compute the instrumentality of the information signal, (2) other reward related regions, such as the nucleus accumbens and dopaminergic midbrain, underlie valence-driven information-seeking, and (3) a network independent from reward processing regions, including dACC, rlPFC and parietal cortex, may encode the drive to reduce uncertainty. These motives can all influence information predictions (rectangle shown in the picture), with a possible integration in the OFC to compute information value. Depending on the value of the information prediction, a decision to seek or avoid information is made. If information is received, information feedback is delivered and the value associated with each motive is updated (dashed lines in the figure). The exact nature of these update signals remains mostly unknown, although limited evidence suggests that updates could be achieved through the computation of a prediction error, such as a general information prediction error (IPE) influencing information prediction, and being modulated by valence (VD-IPE). 


\section{$\underline{\text { References }}$}

Badre, D., Doll, B. B., Long, N. M., \& Frank, M. J. (2012). Rostrolateral prefrontal cortex and individual differences in uncertainty-driven exploration. Neuron, 73(3), 595-607. doi:10.1016/j.neuron.2011.12.025

Bartra, O., McGuire, J. T., \& Kable, J. W. (2013). The valuation system: a coordinate-based meta-analysis of BOLD fMRI experiments examining neural correlates of subjective value. Neuroimage, 76, 412427. doi:10.1016/j.neuroimage.2013.02.063

Bénabou, R. (2016). Mindful Economics: The Production, Consumption, and Value of Beliefs. JOURNAL OF ECONOMIC PERSPECTIVES, 30, 141-164.

Berlyne, D. E. (1957). Uncertainty and conflict: A point of contact between information-theory and behavior-theory concepts. Psychological Review, 329-339.

Berns, G. S., Chappelow, J., Cekic, M., Zink, C. F., Pagnoni, G., \& Martin-Skurski, M. E. (2006). Neurobiological substrates of dread. Science, 312(5774), 754-758. doi:10.1126/science.1123721

Blanchard, T. C., Hayden, B. Y., \& Bromberg-Martin, E. S. (2015). Orbitofrontal cortex uses distinct codes for different choice attributes in decisions motivated by curiosity. Neuron, 85(3), 602-614. doi:10.1016/j.neuron.2014.12.050

Bromberg-Martin, E. S., \& Hikosaka, O. (2009). Midbrain dopamine neurons signal preference for advance information about upcoming rewards. Neuron, 63(1), 119-126. doi:10.1016/j.neuron.2009.06.009

Bromberg-Martin, E. S., \& Hikosaka, O. (2011). Lateral habenula neurons signal errors in the prediction of reward information. Nat Neurosci, 14(9), 1209-1216. doi:10.1038/nn.2902

Bromberg-Martin, E. S., \& Monosov, I. E. (2020). Neural circuitry of information seeking. Current Opinion in Behavioral Sciences.

Brydevall, M., Bennett, D., Murawski, C., \& Bode, S. (2018). The neural encoding of information prediction errors during non-instrumental information seeking. Sci Rep, 8(1), 6134. doi:10.1038/s41598-018-24566-x

Caplin, A., \& Leahy, J. (2001). Psychological Expected Utility Theory and Anticipatory Feelings. The Quarterly Journal of Economics, 116(1), 55-79.

Charpentier, C. J., Bromberg-Martin, E. S., \& Sharot, T. (2018). Valuation of knowledge and ignorance in mesolimbic reward circuitry. Proc Natl Acad Sci $U$ S A, 115(31), E7255-E7264. doi:10.1073/pnas.1800547115

Chib, V. S., Yun, K., Takahashi, H., \& Shimojo, S. (2013). Noninvasive remote activation of the ventral midbrain by transcranial direct current stimulation of prefrontal cortex. Transl Psychiatry, 3, e268. doi:10.1038/tp.2013.44

Cogliati Dezza, I., Cleeremans, A., \& Alexander, W. (2020). Independent and interacting value systems for reward and information in the human brain. bioRxiv.

Cogliati Dezza, I., \& Sharot, T. (in preparation). what drives human infromation-seeking?

Cogliati Dezza, I., Yu, A. J., Cleeremans, A., \& Alexander, W. (2017). Learning the value of information and reward over time when solving exploration-exploitation problems. Sci Rep, 7(1), 16919. doi:10.1038/s41598-017-17237-w

Diederen, K. M. J., \& Fletcher, P. C. (2021). Dopamine, Prediction Error and Beyond. Neuroscientist, 27(1), 30-46. doi:10.1177/1073858420907591

Dubey, R., \& Griffiths, T. L. (2020). Reconciling novelty and complexity through a rational analysis of curiosity. Psychol Rev, 127(3), 455-476. doi:10.1037/rev0000175

Evans, B. M., \& Chi, E. H. (2010). An elaborated model of social search. Information Processing \& Management, 46, 656-678.

Evans, B. M., Kairam, S., \& Pirolli, P. (2009). Do your friends make yousmarter?: An analysis of social strategies in online information seeking. Information Processing \& Management, 46(6), 679-692.

Filimon, F., Nelson, J. D., Sejnowski, T. J., Sereno, M. I., \& Cottrell, G. W. (2020). The ventral striatum dissociates information expectation, reward anticipation, and reward receipt. Proc Natl Acad Sci U $S$ A, 117(26), 15200-15208. doi:10.1073/pnas.1911778117 
Foley, N. C., Kelly, S. P., Mhatre, H., Lopes, M., \& Gottlieb, J. (2017). Parietal neurons encode expected gains in instrumental information. Proc Natl Acad Sci U S A, 114(16), E3315-E3323. doi:10.1073/pnas.1613844114

Frank, M. J., Doll, B. B., Oas-Terpstra, J., \& Moreno, F. (2009). Prefrontal and striatal dopaminergic genes predict individual differences in exploration and exploitation. Nat Neurosci, 12(8), 1062-1068. doi:10.1038/nn.2342

Friston, K., Rigoli, F., Ognibene, D., Mathys, C., Fitzgerald, T., \& Pezzulo, G. (2015). Active inference and epistemic value. Cogn Neurosci, 6(4), 187-214. doi:10.1080/17588928.2015.1020053

Friston, K. J., Lin, M., Frith, C. D., Pezzulo, G., Hobson, J. A., \& Ondobaka, S. (2017). Active Inference, Curiosity and Insight. Neural Comput, 29(10), 2633-2683. doi:10.1162/neco_a_00999

Gershman, S. J. (2018). Deconstructing the human algorithms for exploration. Cognition, 173, 34-42. doi:10.1016/j.cognition.2017.12.014

Gershman, S. J. (2019). Uncertainty and Exploration. Decision, 6(3), 277-286.

Goldman-Rakic P.S., L. M. S., Smiley J.F., Williams M.S. (1992). The anatomy of dopamine in monkey and human prefrontal cortex. In S. E. M. Tuma A.H., Gershon S. (Ed.), Advances in Neuroscience and Schizophrenia. Vienna: Springer.

Golman, R., Hagmann, D., \& Loewenstein, G. (2017). Information avoidance. Journal of Economic Literature, 55(1), 96-135.

Gottlieb, J., Oudeyer, P. Y., Lopes, M., \& Baranes, A. (2013). Information-seeking, curiosity, and attention: computational and neural mechanisms. Trends Cogn Sci, 17(11), 585-593. doi:10.1016/j.tics.2013.09.001

Horan, M., Daddaoua, N., \& Gottlieb, J. (2019). Parietal neurons encode information sampling based on decision uncertainty. Nat Neurosci, 22(8), 1327-1335. doi:10.1038/s41593-019-0440-1

Hunt, L. T., Malalasekera, W. M. N., de Berker, A. O., Miranda, B., Farmer, S. F., Behrens, T. E. J., \& Kennerley, S. W. (2018). Triple dissociation of attention and decision computations across prefrontal cortex. Nat Neurosci, 21(10), 1471-1481. doi:10.1038/s41593-018-0239-5

Iigaya, K., Hauser, T. U., Kurth-Nelson, Z., O'Doherty, J. P., Dayan, P., \& Dolan, R. J. (2020). The value of what's to come: Neural mechanisms coupling prediction error and the utility of anticipation. Sci $A d v, 6(25)$, eaba3828. doi:10.1126/sciadv.aba3828

Iigaya, K., Story, G. W., Kurth-Nelson, Z., Dolan, R. J., \& Dayan, P. (2016). The modulation of savouring by prediction error and its effects on choice. Elife, 5. doi:10.7554/eLife.13747

Jessup, R. K., \& O'Doherty, J. P. (2014). Distinguishing informational from value-related encoding of rewarding and punishing outcomes in the human brain. Eur J Neurosci, 39(11), 2014-2026. doi:10.1111/ejn.12625

Kaanders, P., Juechems, K., O’Reilly, J. X., \& Hunt, L. T. (2021). Dissociable mechanisms of information sampling in prefrontal cortex and the dopaminergic system. Current Opinion in Behavioral Sciences, 41, 63-70.

Kaanders, P., Nili, H., O’Reilly, J. X., \& Hunt, L. T. (2020). Medial frontal cortex activity predicts information sampling in economic choice. bioRxiv preprint. doi:https://doi.org/10.1101/2020.11.24.395814;

Karlsson, N., Loewenstein, G., \& Seppi, D. (2009). The ostrich effect: selective attention to information. $J$ Risk Uncertain 38(2), 95-115.

Kelly, C. A., \& Sharot, T. ((under review)). Deciding what to know: Individual differences in informationseeking.

Kidd, C., \& Hayden, B. Y. (2015). The Psychology and Neuroscience of Curiosity. Neuron, 88(3), 449460. doi:10.1016/j.neuron.2015.09.010

Kobayashi, K., \& Hsu, M. (2019). Common neural code for reward and information value. Proc Natl Acad Sci U S A, 116(26), 13061-13066. doi:10.1073/pnas.1820145116

Kobayashi, K., Lee, S., Filipowicz, A., McGaughey, K., Kable, J. W., \& Nassar, M. R. (2021). Dynamic Representation of the Subjective Value of Information. bioRxiv. 
Kobayashi, K., Ravaioli, S., Baranes, A., Woodford, M., \& Gottlieb, J. (2019). Diverse motives for human curiosity. Nat Hum Behav, 3(6), 587-595. doi:10.1038/s41562-019-0589-3

Ligneul, R., Mermillod, M., \& Morisseau, T. (2018). From relief to surprise: Dual control of epistemic curiosity in the human brain. Neuroimage, 181, 490-500. doi:10.1016/j.neuroimage.2018.07.038

Loewenstein, G. (1987). Anticipation and the Valuation of Delayed Consumption. The Economic Journal, 97(387), 666-684.

Loewenstein, G., \& Molnar, A. (2018). The renaissance of belief-based utility in economics. nature human behaviour.

Lopez-Persem, A., Bastin, J., Petton, M., Abitbol, R., Lehongre, K., Adam, C., . . Pessiglione, M. (2020). Four core properties of the human brain valuation system demonstrated in intracranial signals. Nat Neurosci, 23(5), 664-675. doi:10.1038/s41593-020-0615-9

Matsumoto, M., \& Hikosaka, O. (2007). Lateral habenula as a source of negative reward signals in dopamine neurons. Nature, 447(7148), 1111-1115. doi:10.1038/nature05860

Morris, L. S., Kundu, P., Dowell, N., Mechelmans, D. J., Favre, P., Irvine, M. A., . . . Voon, V. (2016). Fronto-striatal organization: Defining functional and microstructural substrates of behavioural flexibility. Cortex, 74, 118-133. doi:10.1016/j.cortex.2015.11.004

Murayama, K. (2019a). A reward-learning framework of autonomous knowledge acquisition: An integrated account of curiosity, interest, and intrinsic-extrinsic rewards. preprint. doi:10.31219/osf.io/zey4k

Murayama, K. (2019b). A reward-learning framework of autonomous knowledge acquisition: An integrated account of curiosity, interest, and intrinsic-extrinsic rewards. OSFPREPRINTS. doi:10.31219/osf.io/zey4k

Oudeyer, P.-Y. (2018). Computational Theories of Curiosity-driven Learning (G. Gordon Ed. NOVA ed.). Oudeyer, P.-Y., Lopes, M., Kidd, C., \& Gottlieb, J. (2016). Curiosity and Intrinsic Motivation for Autonomous Machine Learning. ERCIM News, 107, 34-35.

Oudeyer, P. Y., \& Kaplan, F. (2007). What is Intrinsic Motivation? A Typology of Computational Approaches. Front Neurorobot, 1, 6. doi:10.3389/neuro.12.006.2007

Padoa-Schioppa, C., \& Conen, K. E. (2017). Orbitofrontal Cortex: A Neural Circuit for Economic Decisions. Neuron, 96(4), 736-754. doi:10.1016/j.neuron.2017.09.031

Pessiglione, M., \& Lebreton, M. (2014). From the Reward Circuit to the Valuation System: How the Brain Motivates Behavior. In T. M. Gendolla G., Koole S. (Ed.), Handbook of Biobehavioral Approaches to Self-Regulation. New York: Springer.

Pierson, E., \& Goodman, N. (2014). Uncertainty and denial: a resource-rational model of the value of information. PLoS One, 9(11), e113342. doi:10.1371/journal.pone.0113342

Schultz, W., Dayan, P., \& Montague, P. R. (1997). A neural substrate of prediction and reward. Science, 275(5306), 1593-1599. Retrieved from https://www.ncbi.nlm.nih.gov/pubmed/9054347

Schulz, E., \& Gershman, S. J. (2019). The algorithmic architecture of exploration in the human brain. Curr Opin Neurobiol, 55, 7-14. doi:10.1016/j.conb.2018.11.003

Schwartenbeck, P., Passecker, J., Hauser, T. U., FitzGerald, T. H., Kronbichler, M., \& Friston, K. J. (2019). Computational mechanisms of curiosity and goal-directed exploration. Elife, 8. doi:10.7554/eLife.41703

Sharot, T., \& Sunstein, C. R. (2020). How people decide what they want to know. Nat Hum Behav, 4(1), 14-19. doi:10.1038/s41562-019-0793-1

Smith, V. D., Rigney, A. E., \& Delgado, M. R. (2016). Distinct Reward Properties are Encoded via Corticostriatal Interactions. Scientific Reports. doi: DOI: 10.1038/srep20093

Story, G. W., Vlaev, I., Seymour, B., Winston, J. S., Darzi, A., \& Dolan, R. J. (2013). Dread and the disvalue of future pain. PLoS Comput Biol, 9(11), e1003335. doi:10.1371/journal.pcbi.1003335

Tomov, M. S., Truong, V. Q., Hundia, R. A., \& Gershman, S. J. (2020). Dissociable neural correlates of uncertainty underlie different exploration strategies. Nat Commun, 11(1), 2371. doi:10.1038/s41467-020-15766-Z

van Lieshout, L. L. F., van den Bosch, R., Hofmans, L., de Lange, F. P., \& Cools, R. (2020). Does dopamine synthesis capacity predict individual variation in curiosity? bioRxiv. 
van Lieshout, L. L. F., Vandenbroucke, A. R. E., Muller, N. C. J., Cools, R., \& de Lange, F. P. (2018). Induction and Relief of Curiosity Elicit Parietal and Frontal Activity. J Neurosci, 38(10), 25792588. doi:10.1523/JNEUROSCI.2816-17.2018

Vellani, V., de Vries, L. P., Gaule, A., \& Sharot, T. (2021). A selective effect of dopamine on informationseeking. Elife.

White, J. K., Bromberg-Martin, E. S., Heilbronner, S. R., Zhang, K., Pai, J., Haber, S. N., \& Monosov, I. E. (2019). A neural network for information seeking. Nat Commun, 10(1), 5168. doi:10.1038/s41467-019-13135-z

Wilson, R. C., Geana, A., White, J. M., Ludvig, E. A., \& Cohen, J. D. (2014). Humans use directed and random exploration to solve the explore-exploit dilemma. J Exp Psychol Gen, 143(6), 2074-2081. doi:10.1037/a0038199

Wu, C. M., Schulz, E., Speekenbrink, M., Nelson, J. D., \& Meder, B. (2018). Generalization guides human exploration in vast decision spaces. Nat Hum Behav, 2(12), 915-924. doi:10.1038/s41562-0180467-4

Zajkowski, W. K., Kossut, M., \& Wilson, R. C. (2017). A causal role for right frontopolar cortex in directed, but not random, exploration. Elife, 6. doi:10.7554/eLife.27430

Zurn, P., \& Bassett, D. S. (2018). On Curiosity: A Fundamental Aspect of Personality, a Practice of Network Growth. Personal Neurosci, 1, e13. doi:10.1017/pen.2018.3 